\title{
Vor verschlossenen Türen - \\ Wie neu sind die Tore und Mauern in lateinamerikanischen Städten?
}

\section{Eine Einführung}

\section{Axel Borsdorf, Innsbruck und Wien}

Auf den ersten Blick erscheinen die ummauerten und mit Toren versehenen Wohnviertel in lateinamerikanischen Städten - sie werden im folgenden als barrios cerrados (geschlossene Viertel) bezeichnet - als neue Elemente im Gefüge der urbanen Zentren des Subkontinents. Als solche wurden sie auch in der bislang dazu erschienenen Literatur bewertet (MEYER \& BäHR 2001; CoY \& PöHLer 2002). Bereits die Tatsache, dass sie trotz spezifischer Arbeiten zur sozialräumlichen Differenzierung lateinamerikanischer Städte der letzten Jahrzehnte (B̈̈Hr \& MERTINS 1995; BorSDORF 1991; ACHILles 1989), oder gar zu den Wohnformen der Oberschicht (АMato 1970; Köster 1995) erst in allerjüngster Zeit eine besondere Beachtung erfahren, zeigt, dass das Phänomen als solches relativ neu zu sein scheint.

Als barrios cerrados werden Wohnquartiere mit mindestens zwei voneinander getrennten Wohnungen oder Häusern bezeichnet, die über eine gemeinsame Infrastruktur verfügen und die von einem Zaun oder einer Mauer eingefriedet und zum öffentlichen Raum durch ein Tor oder eine Schranke abgeschlossen sind. Die Infrastruktureinrichtungen reichen hierbei von der gemeinsamen Grünfläche über Sporteinrichtungen (Fußball, Tennis, Golf etc.), Spielplätze, Schwimmbad bis zum Clubhaus. Ein gesichertes AppartementHochhaus ist erst dann ein barrio cerrado, wenn es gesondert umzäunt und mit einem Tor versehen ist sowie über Gemeinschaftseinrichtungen verfügt.

Die wenigen bislang vorliegenden Studien belegen durchwegs, dass die ersten Straßensperrungen und/ oder die Ummauerung bestehender Viertel in den 1970er Jahren aufkamen (PöHLER 1999; MEYER \& BäHR 2001) und die ersten auf dem Reißbrett entworfenen barrios cerrados zehn Jahre später entstanden. Seither - so scheint es - hat die lateinamerikanische Stadt ein neues Entwicklungsstadium erreicht, das mit einer Verfeinerung des sozialräumlichen Segregationsmusters und der Dispersion von Strukturelementen einhergeht, die zuvor räumliche Konzentrationstendenzen aufwiesen (Industrie, großflächiger Handel, hochwertige Dienstleistungen). Die neue Tendenz zur Fragmentierung der Stadtstruktur wird häufig auf die ökonomische Transformation, bzw. die Hinwendung zu neoliberalen Wirtschaftsstrategien, und auf die zuneh- mende Globalisierung zurückgeführt (vgl. GORENSTEIN \& Bustos 1998). Da die ersten barrios cerrados zeitgleich mit der Globalisierung und Transformation auftraten, scheint diese These auch plausibel.

Ebenso naheliegend ist es, die neuen Viertel als Kopien nordamerikanischer gated communities zu verstehen, wobei das Vorbild sozusagen im Rahmen eines globalen Diffusionsprozesses einer in den Vereinigten Staaten entstandenen Produkt-Innovation nach Lateinamerika importiert worden sei. Dieser Einschätzung liegen u.U. Erfahrungen mit solchen Produkten aus dem europäischen Raum zugrunde, wo tatsächlich derartige Erschließungen auf nordamerikanische Investoren zurückgehen.

Folgerichtig orientieren sich die ersten Versuche zur Typisierung der barrios cerrados an der bekannten Klassifikation von BlaKely \& SNyder (1997) oder übernehmen zumindest das Prinzip der Zuweisung zu verschiedenen Sozialschichten als Ordnungsprinzip (Evangelisti 2000; Borsdorf 2000; Borsdorf 2002a).

Im folgenden soll jedoch gezeigt werden, dass eine solche Erklärung zu kurz greift und in der logischen Folge auch die Frage der Typisierung neu bedacht werden muss. Die in der bisherigen Literatur zu wenig beachtete Tatsache, dass die Umzäunung von Wohnvierteln nicht allein auf die Ober- und Mittelschicht beschränkt ist, sondern in vielen Städten die Mehrheit der barrios cerrados der Unter- und unteren Mittelschicht zuzuordnen ist, weist bereits darauf hin, dass sie nicht allein als Wohnform von "los que ganaron» (Svampa 2001, im Titel. Zu deutsch: «die, die gewonnen haben"), also den Gewinnern der Transformation und Globalisierung zu deuten sind. Auch das Faktum der überaus raschen Verbreitung in allen lateinamerikanischen Staaten und ihre Anlage in Städten aller Größenordnungen führt zur Frage, ob die Tendenz zur Abschottung von der Außenwelt oder, anders formuliert, der Dualismus von Außen- und Innenwelt, und ob Mauern und Tore nicht durchaus der lateinamerikanischen Kultur und Tradition entsprechen und durch die Transformation und Globalisierung nur eine Mutation erfahren haben.

Zumindest für den spanischsprachigen Teil Lateinamerikas gilt, dass mit wenigen Ausnahmen die dort angelegten Städte nach außen hin offen, d.h. nicht ummauert, nach innen dagegen ausgesprochen 
geschlossen waren. Die Wohngebäude - AtriumHäuser mit geschlossenen Innenhöfen (patios) - waren zur Strasse hin durch ein schweres Holztor verschlossen, bis Mitte des 18. Jahrhunderts und oft darüber hinaus waren die Fenster klein und uneinsehbar hoch in der zur Straße gelegenen Mauer angebracht (Foto 1). Dafür wird zumeist das spanische Prinzip der Kühlung durch Mauerspeicherung (BORSDORF \& STADEL 1997: 18) verantwortlich gemacht, das erst später vom portugiesischen Prinzip der Kühlung durch Luftzug (Querlüftung über große Fenster) abgelöst wurde. Das Kühlungsprinzip ist jedoch mehr eine Folge des Bedürfnisses der Bewohner dieser Häuser nach Wahrung der familiären Intimsphäre. Dass darin starke Segregationstendenzen wirksam waren, wird auch daran deutlich, dass nicht nur die Hausräume, sondern auch die hintereinander geschachtelten Innenhöfe nach Familienstellung und Geschlecht zugeordnet waren: Die Welt der Frauen im Küchen- und Gartenpatio war streng von der der Männer getrennt, und ein Gast erhielt niemals Zugang zum zweiten oder dritten Hof.

Diese Patio-Häuser waren aber jeweils eine Wohneinheit und entsprachen oder entsprechen daher nicht der Definition von barrios cerrados. Dennoch gab es auch in der Kolonialstadt bereits solche geschlossene, nur den Bewohnern zugängliche Viertel. Die «Klosterstadt» Santa Catalina in Arequipa, das Jesuitenseminar in Tepotzotlán, das Hospitolio Cabañas in Guadalajara (das freilich erst um die Jahrhundertwende 19.-20. Jh. entstand), sind Beispiele für Stadtviertel von beachtlicher Größe, die Frauen, Witwen, Semina-

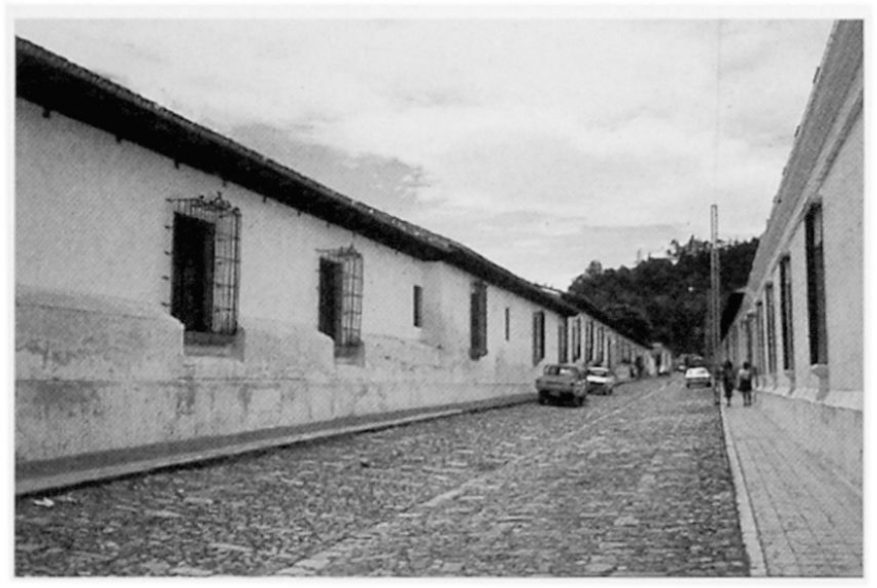

Foto 1: Strasse in Antigua Guatemala: Der öffentliche Raum ist vom privaten strikt getrennt.

Urban road in Antigua Guatemala: Public and private spcae are strictly separated from each other.

Rue urbaine à Antigua Guatemala: l'espace public est strictement séparé de l'espace privé.

Foto: A. BORSDORF risten und Waisen vorbehalten waren. Sie entsprechen in vielen den Klöstern und sind auch häufig monastische Gründungen. Aber auch das Kloster kann als Vorläufer des barrio cerrado verstanden werden.

Im 19. Jahrhundert entstanden am Rand mancher Städte sogenannte company towns, die den Angestellten und Arbeitern von Bergwerk- und Industrieunternehmen vorbehalten waren, extrem segregiert (spezifische Angestellten- und Arbeitersiedlungen) und nicht selten eigens ummauert und mit Schranken versehen waren.

Echte Vorläufer der barrios cerrados sind jedoch die Gängeviertel, die Ende des 19. Jahrhunderts in den Großstädten Lateinamerikas aufkamen. In Chile wurden sie als conventillos (zu deutsch: Klösterchen) bezeichnet, in Peru als tugurios, in Mexiko als vecindades (zu deutsch: Nachbarschaften). Sie entstanden zunächst in den von der Oberschicht aufgegebenen Atrium-Häusern, die nun zimmerweise an die einwandernde Landbevölkerung vermietet oder verkauft wurden. Damit wurde das Einfamilien-Patio-Haus zum Mehrhaushalte-Wohngebäude, das zur Strasse per schwerem Holztor gesichert auch in der Grundstruktur bereits den heutigen barrios cerrados der Unterschicht entspricht (Foto 2). Derartige Strukturen wurden auch eigens angelegt, als die alte Bausubstanz nicht mehr ausreichte. In Chile entstanden derartige Strukturen vor allem in den 1920er und 1930er Jahren und wurden dann mit der Bezeichnung cité versehen (vgl. WILHelmy \& Borsdorf 1985: 155f., die Karte eines so überbauten ganzen Wohnblocks findet sich auf Seite 156). Hidalgo (1999) und Rovira (2002:354) haben auf die Verwandtschaft dieser Viertel mit den heutigen barrios cerrados hingewiesen und belegt. dass bereits 1888 rechtliche Regelungen für die Eigentumsverhältnisse in conventillos und cités erlassen wurden.

In Mexiko wurden vecindades noch bis zum Beginn der 1950er Jahre angelegt. Anders als in Chile, wo es beim Prinzip des ebenerdigen Patio-Hauses blieb und die Wohnzellen der cités aus einem Multifunktionsraum und einer vorgelagerten, offenen Küchenzelle bestanden, wobei sich die Bewohner die zentral gelegenen Toiletten und Wasserbecken teilen mussten. haben die neueren mexikanischen vecindades bereits einen Wasser- und Kanalisationsanschluss in den Wohnungen. Sie sind bis zu dreistöckig als Stock werkeigentum angelegt. Zweimal zwei einander gegenüberliegende Wohnungen sind über eine zentrale, zum Himmel offene Treppenkonstruktion miteinander verbunden. Zur Straße hin sind sowohl die vecindades als auch die conventillos und tugurios über eine schwere Tür verschlossen.

Das Tor des conventillo schützte und schützt die vecindad vor der Aussenwelt, d.h. vor der Gewalt 


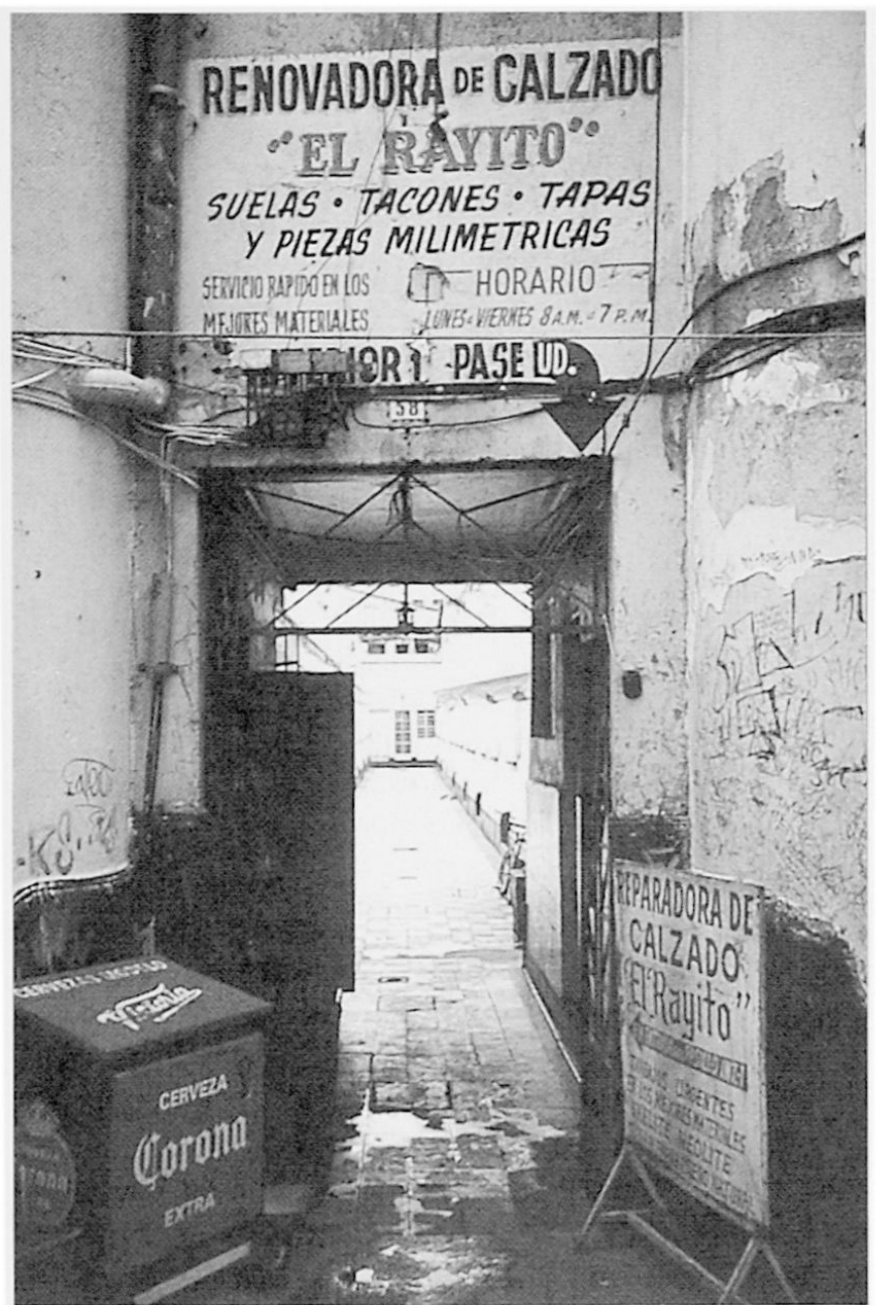

Foto 2: Vecindad in Mexiko-Stadt: Ein einziges Tor verschliesst den Zugang zu den Wohneinheiten.

"Vecindad" in Mexico City: The complex can only be entered through the main gates.

"Vecindad" à Mexico-Cité: L'entrée unique verrouille l'accès aux unités d'habitation.

Foto: A. BORSDORF

von Einzelpersonen, in gewisser Weise aber auch vor staatlicher Willkür, weil jeder Eingriff von der Nachbarschaft bemerkt wird, die dann kollektiv reagieren kann. Es versteht sich fast von selbst, dass eine solche Wohnform sowohl in Zeiten staatlicher Omnipotenz (z.B. unter Militärregierungen) als auch bei einem Rückgang der Kontrollfunktion des Staates (also im Neoliberalismus) dem Bedürfnis der Menschen nach Sicherheit und nachbarschaftlicher Hilfe entgegenkommt. So lassen sich die heutigen barrios cerrados der Unterschicht auf die cités und vecindades zurückführen, denen sie in ihrer Grundstruktur (vgl. die Abb. des barrio cerrado «La Punta»/Lima in BORSDORF 2002a: 587) verblüffend ähneln.

Man hat in diesem Zusammenhang von einem Rückfall des lateinamerikanischen Urbanismus ins Mittelalter gesprochen (Geraiges DE Lemos et al. 2002, im Titel), tatsächlich erinnern manche Bauformen an die Ummauerung mittelalterlicher Städte (vgl. S. 303, Foto 2 , in diesem Heft). Mit diesen Beispielen sollte gezeigt werden, dass eine solche Sicht falsch ist. Nicht nur, dass die Urbanisierung Lateinamerikas im modernen Sinn erst mit der Neuzeit, d.h. der Conquista begann, auch existierten selbst dort, wo Städte befestigt wurden, nirgends in der Neuen Welt jene Rechtsgrenzen, für die die Stadtmauer im europäischen Mittelalter stand. Auch die Einfriedung von Wohnvierteln schafft keine neuen Rechtsräume im Sinne eines «Bürger und Bauer scheidet die Mauer», insofern ist der Vergleich mit den mittelalterlichen Stadtmauern völlig unangebracht. Stattdessen ist festzustellen, dass Mauern zumindest im hispanischen Teil Amerikas keineswegs konstituierende Elemente der Stadtstruktur waren. In diesem Zusammenhang sei kurz auf das Kuriosum hingewiesen, dass in den letzten Dekaden auch Mauern entstanden sind, die nicht die Aufgabe haben, die Stadt einzufrieden, sondern im Gegenteil, das Land vor dem städtischen Wachstum zu schützen.

Auch die barrios cerrados der Oberschicht haben, zumindest, was den Typ des lifestyle-barrio (BorsDORF 2000 und 2002a) betrifft, Vorgänger in früheren Entwicklungsphasen der lateinamerikanischen Stadt. Viele Autoren von monographischen Einzelstudien kommen zum gleichen Ergebnis, indem sie betonen, dass diese Viertel ursprünglich in Anlehnung an einen Country- oder Golfclub entstanden, dann allmählich überbaut, aber erst in den 1980er Jahren mit einer Mauer und einem zentralem Eingangstor versehen wurden. Die Urform eines solchen "Club de Campo" ist zweifelsohne der Club Campestre de la Ciudad de México, auch als Churubusco Golf Club bekannt. Seine Genese entspricht fast dem Stereotyp einer aus einem anderen Kulturraum nach Lateinamerika übertragenen Lebensform. Um 1900 trafen sich einige Engländer, um in der freien Natur (in diesem Fall in Los Pinos) ihrer Leidenschaft des Golfspiels zu frönen. 1905 wurde das Gelände des heutigen Golfclubs erworben (heute $72 \mathrm{ha}$ ), damals waren es noch Viehweiden weit außerhalb der Stadt, und darauf ein 18-Loch-Golfplatz sowie ein repräsentatives Clubhaus mit Ballsaal angelegt, das in der städtischen Gesellschaft - vor allem bei Europäern - bald für Anlässe aller Art genutzt wurde. Aus den Einnahmen konnten die Gründer in der Folge stattliche Villen auf dem Gelände finanzieren. Heute befinden sich auf dem Gelände des «Fraccionamento Cerrado Club de Golf de Churubusco" (amtliche Bezeichnung dieses barrio cerrado) 19 Villen, ein Clubhaus mit ausgedehnten Gesellschaftsräumen, ein 18- und ein 9-Loch-Golfplatz, Tennisplätze, eine Turnhalle, ein Schwimmbad und ein Teich für Paddelboote. 
In diesem Fall lag das Schwergewicht noch auf der Infrastruktur für den gemeinsam ausgeübten Sport. In vielen anderen lateinamerikanischen Städten entstanden die sogenannten countries in den 1950er und 60er Jahren, als sich nach dem Ende des Zweiten Weltkriegs die Sympathie der Oberschicht eindeutig dem nordamerikanischen Vorbild zuzuneigen begann. Dennoch darf nicht übersehen werden, dass die Clubes de Campo zumindest ursprünglich nicht ausschließlich Kopien englischer oder nordamerikanischer Lebensstile waren. Sie entsprachen auch in der Zeit stürmischer Industrialisierung (importsubstituierende Industrialisierung), zunehmender Motorisierung und Umweltverschmutzung (Smog und Lärm) der Sehnsucht der Menschen nach Natur, Ruhe, reiner Luft und einem dieser arkadischen Umgebung angepassten Lebensstil. Die Städtefeindlichkeit der Amerikaner (BORSDORF 1989; HolzNer 1985), die nordamerikanische Sehnsucht nach Arkadien, die sich bei den Agrarians am deutlichsten artikulierte (BORSDORF 2002b), und schließlich das nordamerikanische Ideal der Cowboy-Figur treffen hier auf den Stadtüberdruss einer lateinamerikanischen Oberschicht, ihre Orientierung an nicht lateinamerikanischen Lebensstilen, ihren Wunsch nach Exklusivität und die Stilisierung und Idealisierung des Gaucho (in Argentinien) bzw. Huaso (in Chile), wo solche Clubs durchaus auch Gaucho-Club oder Huaso-Club heißen können. Die rapide Zunahme solcher Viertel seit den 1980er Jahren ist bis zu einem gewissen Grad als Resultat der kulturellen Globalisierung (Hoffmann 2002), d.h. der Ausbreitung des American way of life, zu deuten, wobei sie freilich infolge der Existenz ähnlicher, aber autochthon-lateinamerikanischer Idealisierungen des Landlebens in vielen Ländern Lateinamerikas einen aufnahmefähigen Boden fand.

Damit reduziert sich der primäre Einfluss der Transformation und Globalisierung auf jene geschlossenen Viertel der Oberschicht, die nicht auf das Selbstverständnis

Globale Ebene

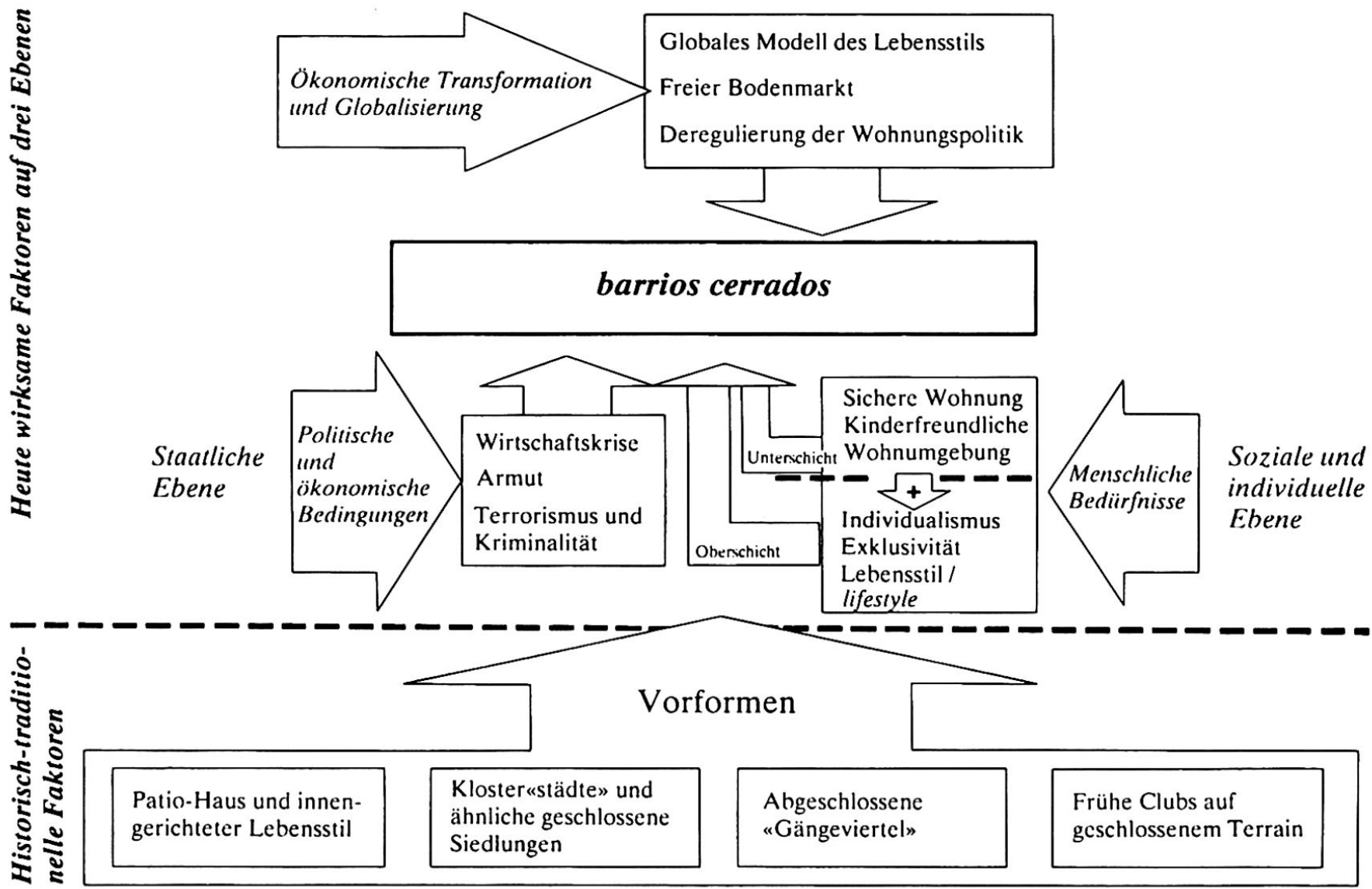

Abb. 1: Kausalfaktoren der Entstehung der barrios cerrados in Lateinamerika Factors contributing to the establishment of "barrios cerrados" in Latin America Les facteurs explicatifs de l'émergence des "barrios cerrados» en Amérique latine Entwurf: A. Borsdorf; Graphik: L. BAUMANN 
als Country-Club (oder Chacra-Club in Argentinien) bauen sowie auf die barrios cerrados der Mittelschicht. Dennoch ist nicht abzustreiten, dass die Tendenz zur Abschottung von Wohnvierteln auch in der Unterschicht und in der einen spezifischen Lebensstil pflegenden Oberschicht ebenfalls durch die beiden miteinander in Beziehung stehenden Prozesse von Transformation und Globalisierung beeinflusst wird. Die Betonung der Privatsphäre bei gleichzeitigem Misstrauen gegenüber der Gesamtgesellschaft ist jedenfalls in allen Gesellschaftsschichten Lateinamerikas verbreitet.

Abb. 1 stellt das Ursachengefüge für die Entstehung von abgeschlossenen Vierteln in der spanisch-amerikanischen Stadt in vereinfachter Form dar.

Die in diesem Heft vorgelegten Beiträge dokumentieren eine am Institut für Stadt- und Regionalforschung der Österreichischen Akademie der Wissenschaften (ISR) durchgeführte Tagung zu den barrios cerrados in Lateinamerika, die dem Erfahrungsaustausch in diesem neuen Forschungsfeld diente. An dieser Tagung haben nahezu alle Forscher im deutschen Sprachraum, die sich derzeit mit dem Phänomen von barrios cerrados in Lateinamerika beschäftigen, als Redner oder Diskussionspartner teilgenommen. Mit Peter MARCUSE, einem der bedeutenden Theoretiker der Segregationsforschung in den USA fand einige Tage später im ISR ein Gespräch statt, aus dem einige der Beiträge weitere Anregungen schöpfen.

Der Praxis an einer Forschungsfront entsprechend, war nicht zu erwarten, dass sich ein homogenes Bild ergeben würde. Bei allen Unterschieden der Perspektive und Methode entsteht erstaunlicherweise dennoch ein relativ rundes Bild dieses Phänomens, wobei sich die Einschätzung der verursachenden Faktoren von Fall zu Fall unterscheidet. Fischer und Parnreiter betonen die Bedeutung von Transformations- und Globalisierungsprozessen für die Verschärfung der sozialen Kontraste und die Zunahme der räumlichen Segregation, KanITSCHEIDER stellt ihre Untersuchung für die Agglomeration Mexiko-Stadt unter den erkenntnisleitenden Gesichtspunkt der Segregationstheorie, CoY und PöHLER beziehen sich mit ihren Beispielen auf den lusoamerikanischen Kulturraum. Die dort geschilderten Megaprojekte finden sich auch in Buenos Aires, wo JANOSCHKA seine Studie angelegt hat, wobei er auf der Grundlage qualitativer sozialwissenschaftlicher Methodik die Akteure selbst in die Analyse einbezieht. In der kleinen und sehr konservativen Hauptstadt Ecuadors würde man eine starke Präsenz von barrios cerrados vielleicht weniger vermuten. KOHLER zeigt in ihrer Studie, dass dies keineswegs so ist, wobei sie anhand von zwei Rechtsformen, die zugleich sozioökonomisch zuzuordnen sind, darlegt, dass sich die Viertel der Rei- chen noch nach dem Muster der polarisierten Stadt, die geschlossenen Viertel der Unter- und unteren Mittelschicht sich jedoch bereits nach dem neueren Muster der fragmentierten Stadt verteilen.

Dies mündet schließlich in die Frage, inwieweit die Entwicklungsprozesse der letzten dreißig bis zwanzig Jahre die Stadtstruktur lateinamerikanischer Städte so weit veränderten, dass das herkömmliche «Modell» der lateinamerikanischen Stadt, wie es in verschiedenen Versuchen vor rund zwanzig Jahren von BäHR, Borsdorf, Gormsen, Griffin \& Ford und Mertins (Quellen in: BORSDORF, BäHR \& JANOSCHKA in diesem Heft) vorgelegt wurde, heute verändert werden muss. Dass dies offenbar nötig ist, belegen neue Versuche von MEYER \& BÄHR (2001), BORSDORF (2002b) und JANOSCHKA (2002). Um eine neuerliche Verwirrung durch die nahezu gleichzeitige Vorstellung doch relativ unterschiedlicher Modelle zu vermeiden, unternehmen die Autoren BäHR, Borsdorf und JaNOSCHKA im letzten Beitrag einen Versuch zur Entwicklung eines einzigen Modells, das die an unterschiedlichen Fallstudien gewonnenen Erkenntnisse verbindet.

\section{Dank}

Diese Arbeit wurde finanziert durch den Fonds zur Förderung wissenschaftlicher Forschung (Nummer P 14883).

\section{Literatur}

Achilles, G.W. (1989): Strukturwandel und Bewertung sozial hochrangiger Wohnviertel in Rio de Janeiro. Die Entwicklung einer brasilianischen Metropole unter besonderer Berücksichtigung der Stadtteile Ipanema und Leblon. - = Tübinger Geographische Studien 104, Tübingen.

Amato, P.W. (1970): Elitism and Settlement-Patterns in the Latin American City. - In: Journal of the American Institute of Planners 36: 96-105.

BäHR, J. \& G. MerTins (1995): Die lateinamerikanische Großstadt. Verstädterungsprozesse und Stadtstrukturen. - = Erträge der Forschung 288, Darmstadt.

Barden, S., Geiss, S. \& M. JanoschKa (2000): Wohnpark Mühlendorf, Teltow. Marktchancen, Zielgruppen und Konsequenzen für die Projektentwicklung. Standortgutachten im Auftrag der Mühlendorf $\mathrm{GmbH}$ \& Co. KG, Berlin.

Blakely, E.J. \& M.G. SNyder (1997): Fortress America. Gated Communities in the United States. - Washington, D.C.: Brookings Institution Press.

BorsDorf, A. (1989): Crisis urbana in der Neuen Welt? Interpretationen und Lösungsansätze aus kulturgenetischer Sicht. - In: Tübinger Geographische Studien 103: 99-117.

Borsdorf, A. (1991): Stadtkrise oder Kulturkrise? 
Reflexionen über die Bedeutung der Stadt für Kultur und Entwicklung in Lateinamerika. - In: KoHLHEPP, G. (Hrsg.): Lateinamerika - Umwelt und Gesellschaft zwischen Krise und Hoffnung. - In: Tübinger Geographische Studien 107: 133-156.

Borsdorf, A. (2000): Die Condominios von Santiago de Chile als Beispiele sozialräumlicher Segregationstendenzen von Ober- und Mittelschicht in lateinamerikanischen Städten. - In: Peripherie. Zeitschrift für Politik und Ökonomie in der Dritten Welt 20, 80: 25-40.

Borsdorf, A. \& C. Stadel (1997): Ecuador in Profilen. - = Inngeo, Innsbrucker Materialien zur Geographie 3, Innsbruck.

Borsdorf, A. (2002a): Barrios cerrados en Santiago de Chile, Quito y Lima: tendencias de la segregación socio-espacial en capitales andinas. - In: CABRALES Barajas, L.F. (Hrsg.): Latinoamérica: países abiertos, ciudades cerradas. - Guadalajara, Paris: Universidad de Guadalajara, UNESCO: 581-610.

Borsdorf, A. (2002b): Der Alte und der Neue Süden. Geographisches Tagebuch 6.-22.4.2001. - In: BORSDORF, A., Draxlbauer, M. \& W. Zacharasiewicz (Hrsg.): Sites of Memory and Collective Identities. - In: Inngeo 11, Innsbruck: 5-56.

Evangelisti, B. (2000): Räumliche Segregation, Gated Communities/Condominios in Santiago de Chile. Unveröffentlichte Diplomarbeit am Institut für Geographie, Universität Wien.

Geraiges de Lemos, A.I., Capuano Scarlato, F. \& R.P. PÉrez Machado (2002): Las metrópolis del Cono Sur: $\mathrm{O}$ ritorno à cidade medieval: os condominios fechados da metrópole paulistana. - In: CABRALEs BARAJAS, L.F. (Hrsg.) : Latinoamérica: países abiertos, ciudades cerradas. - Guadalajara, Paris: Universidad de Guadalajara, UNESCO: 217-235.

Gorenstein, S. \& R. Bustos Cara (Hrsg.) (1998): Ciudades y regiones frente al avance de la globalización. Bahía Blanca: Universidad Nacional del Sur.

Hidalgo, R. (1999): Continuidad y cambio en un siglo de vivienda social en Chile (1892-1998). Reflexiones a partir del caso de la Ciudad de Santiago. - In: Revista Geográfica Norte Grande 26: 69-77.

HofFMANN, S. (2002): The clash of globalization. - In: Foreign Affairs 81, 4: 104-115.

Holzner, L. (1985): Stadtland USA. Zur Auflösung und Neuordnung der US-amerikanischen Stadt. - In: Geographische Zeitschrift 73, 4: 191-205.

JANOSCHKA, M. (2002): Urbanizaciones privados en Buenos Aires: Hacia un nuevo modelo de ciudad latinoamericana? - In: CABRALES BARAJAS, L.F. (Hrsg.): Latinoamérica: países abiertos, ciudades cerradas. - Guadalajara, Paris: Universidad de Guadalajara, UNESCO: 287-318.

KöSTER, G. (1995): Bevölkerungsstruktur, Migrationsverhalten und Integration der Bewohner von Mittelund Oberschichtsvierteln in der lateinamerikanischen
Stadt. Das Beispiel La Paz (Bolivien). - = Aachener Geographische Arbeiten 30, Aachen.

MEYER, K. \& J. BÄHR (2001): Condominios in Greater Santiago de Chile and their Impact on the Urban Structure. - In: Die ERDE 132, 3: 293-321.

PöHLER, M. (1999): Zwischen Luxus-Ghettos und Favelas. Stadterweiterungsprozesse und sozialräumliche Segregation in Rio de Janeiro: Das Fallbeispiel Barra da Tijuca. - = Kleinere Arbeiten aus dem Geographischen Institut der Universität Tübingen 21, Tübingen.

Rovira, A. 2002: Los barrios cerrados de Santiago de Chile: En busca de la seguridad y la privacidad perdidas. - In: Cabrales Barajas, L.F. (Hrsg.) : Latinoamérica: países abiertos, ciudades cerradas. - Guadalajara, Paris: Universidad de Guadalajara, UNESCO: 351-369.

SVAMPA, M. (2001): Los que ganaron. La vida en los countries y barrios privados. - Buenos Aires: Editorial Biblos.

Wilhelmy, H. \& A. Borsdorf (1985): Die Städte Südamerikas. Band 2: Die urbanen Zentren und ihre Regionen. - Berlin, Stuttgart: Gebrüder Bornträger.

\section{Zusammenfassung: Vor verschlossenen Türen - Wie neu sind die Tore und Mauern in lateinamerikani- schen Städten? Eine Einführung}

Die Städte Lateinamerikas haben in den letzten Dekaden ein neues Gesicht bekommen. Darin stechen die ungewohnten Mauern und Zäune, mit denen Straßen abgesperrt werden und hinter denen sich kleinere oder größere Wohnkomplexe verbergen, in besonderer Weise hervor. Manche Autoren halten diese abgeriegelten Bezirke für eine Kopie nordamerikanischer gated communities, andere bewerten die neuen Mauern als Rückfall in das Mittelalter.

Diesen Auffassungen muss entgegengehalten werden, dass die Tendenz zur Abschottung in lateinamerikanischen Städten eine lange Tradition hat. Bereits die frühen Patio-Häuser bedeuteten eine radikale Zäsur zum öffentlichen Raum, dem sie sich nur mit einer geschlossenen Mauer und nur wenigen kleinen Gebäudeöffnungen zeigten. Andere Beispiele verdeutlichen, dass auch in der traditionellen Stadt ganze Wohnkomplexe abgeriegelt wurden, so etwa auch die Gängeviertel vom conventillo-Typ, die bis in die 1950er Jahre angelegt wurden.

Die lateinamerikanischen barrios cerrados haben demnach durchaus auch autochthone Wurzeln, wenngleich die ökonomische Transformation und Globalisierung weitere wichtige Ursachen für das Aufkommen dieser neuen Wohn- und Lebensform darstellen. 
Summary: Looking at Closed Doors - how new are gates and walls in Latin American cities?

An introduction to the special issue

The face of urban centers in Latin America has changed during the last decades. Particularly the erection of fences and walls to close streets and encircle residential complexes varying in size emphasise this change. Some researchers see closed-off districts as copies of the North American gated communities, others interpret the changes as a relapse to medieval times.

However, it should be remembered that the tendency to shield private space behind walls is nothing new in Latin American cities. Originally, the patio house was a radical break from the traditional openness to public space, a thick wall with a few small openings shielding the house from the street. Before the introduction of gated communities, whole districts in the traditional city were enclosed (e.g. conventillos, tugurios and vecindades, which were constructed until the 1950's).

Thus, the barrios cerrados in Latin America clearly have autochthonous roots. Nevertheless, economic transformation and globalisation have certainly contributed greatly to the emergence of these new residential and living forms.

Résumé: Devant des portes fermées - Portes et murs dans les villes latino-américaines ont-ils un caractère nouveau? Une introduction à la thématique

Les villes latino-américaines ont changé de visage au cours des dernières décennies. Les murs et clôtures inhabituelles, qui barrent l'accès routier et derrière lesquels se cachent de petits et grands complexes résidentiels, se détachent tout particulièrement dans le paysage urbain. Certains auteurs prennent ces ensembles verrouillés pour une imitation des gated communities nordaméricaines; d'autres considèrent les nouveaux murs comme un retour au Moyen-Âge.

Ces conceptions doivent être contrées par le fait que la tendance à la ségrégation repose sur une longue tradition dans les villes sudaméricaines. Déjà les anciennes maisons à patio ont constitué une césure radicale au regard de l'espace public, face auquel elles n'offrirent qu'un mur fermé et seulement quelques petites ouvertures. D'autres exemples montrent clairement que dans la ville traditionnelle des complexes entiers d'habitations étaient également verrouillés; ce fut le cas des quartiers de type conventillo, construits jusque dans les années 1950.

Les barrios cerrados latino-américains ont ainsi des racines entièrement autochtones, quoique la transformation économique et la mondialisation aient été des causes profondes de l'apparition de cette nouvelle forme de vie et d'habitation.

\section{Didaktische Hinweise}

- Worin unterscheiden sich die barrios cerrados von den aus Nordamerika bekannten gated communities?

- Welche kulturellen und soziopolitischen Gründe führten in Lateinamerika zu Vorformen abgeschlossenen Wohnens?

- Welche Dimensionen von Exklusion und Segregation sind in der heutigen lateinamerikanischen Stadt neu?

- Wo finden sich Abschliessung und Binnenorientierung in Städten anderer Kulturräume? Wie werden sie gehandhabt? Warum?

- Was sagt ein Vergleich von «Gängevierteln» im vorindustriellen Europa und in Lateinamerika bezüglich Konvergenz und Divergenz?

Prof. Dr. Axel Borsdorf, Institut für Geographie, Universität Innsbruck, Innrain 52, A-6020 Innsbruck; Institut für Stadt- und Regionalforschung, Österreichische Akademie der Wissenschaften, Postgasse 7/4/2, A-1010 Wien.

e-mail: axel.borsdorf@uibk.ac.at

Manuskripteingang/received/manuscrit entré le 11.9.2002 